\title{
Line-probe assay and molecular typing reveal a potential drug resistant clone of Mycobacterium tuberculosis in Ethiopia
}

\author{
Shiferaw Bekele ${ }^{1,2,4 \dagger}$, Yohannes Derese ${ }^{1 \dagger}$, Elena Hailu', Adane Mihret ${ }^{1}$, Kifle Dagne $^{2}$, Lawrence Yamuah',
} Tsegaye Hailu', Samuel Ayele ${ }^{1}$, Demissew Beyene ${ }^{1}$, Stefan Berg ${ }^{3+}$ and Abraham Aseffa ${ }^{1,4^{*}+}$ (D)

\begin{abstract}
Background: Antimicrobial resistance is a global concern of increasing significance. Multidrug resistant tuberculosis (MDR-TB) is spreading worldwide. It is important to monitor trends of antimycobacterial resistance. This is particularly true for high TB burden countries such as Ethiopia where disproportionally less drug sensitivity data are reported from.

Methods: The prevalence of drug resistance was assessed with the line probe assay GenoType MTBDRplus in a set of $161 \mathrm{M}$. tuberculosis strains that were selected from four common lineages and sub-lineages previously identified in Ethiopia. Most of the tested M. tuberculosis isolates had been genotyped by established Spoligotyping and MIRU-VNTR typing methods.

Results: The proportion of MDR-TB among the isolates was 3.1\%. Mono-resistance was $1.2 \%$ to rifampicin and $4.3 \%$ to isoniazid, and resistance to either of the two first line drugs was $8.7 \%$. Strains of Lineage 4 had the highest resistance rate (13.6\%) followed by Lineage 3 (4.9\%). None of the isolates representing Lineages 1 and Lineage 7 were drug resistant. Multidrug resistance among pulmonary TB and TB lymphadenitis clinical isolates was 2.8 and 3. $7 \%$, respectively. Drug resistance of strains carrying the most prevalent spoligotype in Ethiopia - SIT149 - was further explored. Stratification by MIRU-VNTR identified one genotype with a high rate of drug resistance against Rifampicin and Isoniazid and circulation of a potential MDR-TB clone is proposed.

Conclusion: Although the strain selection was not fully randomized, the overall M. tuberculosis drug resistance rate in this strain set was $8.7 \%$ while the rate of MDR was $3.1 \%$. In parallel, we identified a sub-lineage that showed a high rate of resistance to both rifampicin and isoniazid. These resistant strains may belong to a clone of $M$. tuberculosis that is circulating in the highlands of Ethiopia.
\end{abstract}

Keywords: Mycobacterium, Tuberculosis, Drug resistance, Rifampicin, Isoniazid, MDR-TB, Genotype, Pulmonary, Cervical lymphadenitis, Clone

\section{Background}

Multi-drug resistant Mycobacterium tuberculosis (MDR-TB) (resistant to at least both isoniazid and rifampicin) is an increasing threat to all efforts to bring down the prevalence of tuberculosis (TB) worldwide through control strategies such as the Directly Observed

\footnotetext{
* Correspondence: aseffaa@gmail.com

†Shiferaw Bekele, Yohannes Derese, Stefan Berg and Abraham Aseffa contributed equally to this work.

${ }^{1}$ Armauer Hansen Research Institute, Jima Road, Addis Ababa, Ethiopia

${ }^{4}$ Present address: J. Craig Venter Institute, Rockville, MD, USA

Full list of author information is available at the end of the article
}

Treatment, Short-Course (DOTS) program. Recent numbers from the WHO suggest that MDR-TB can be as high as $5 \%$ globally, is present in most affected countries, and is more prevalent among HIV-infected patients [1]. Although resistant strains have been suggested to lose fitness and thus be less transmissible than strains susceptible to TB drugs [2], MDR-TB is on the rise globally and is contributing to higher mortality rates from the disease [3]. MDR-TB strains can be identified with culture on drug containing selective media as well as through molecular typing

(C) The Author(s). 2018 Open Access This article is distributed under the terms of the Creative Commons Attribution 4.0 International License (http://creativecommons.org/licenses/by/4.0/), which permits unrestricted use, distribution, and 
such as with Line probe assays (that recognize specific chromosomal mutations) [4]. Molecular typing techniques on culture isolates allow to pinpoint specific mutations causing resistance in $M$. tuberculosis for both therapeutic guidance and surveillance of drug resistance patterns [5]. In addition, epidemiological information on transmission routes and relative prevalence of circulating $M$. tuberculosis lineages and sub-lineages in a defined geographic region can be generated through such DNA-based typing techniques.

World-wide genotyping efforts have revealed seven major M. tuberculosis lineages (Lineages 1-7) that are established in humans of which two, Lineages 5 and 6 , were more commonly known as Mycobacterium africanum. The geographical distribution varies between these lineages; Lineage 4 (L4) is the dominating lineage in Europe and the Americas while Lineages 1, L2 and L3 are more prevalent in Africa and Asia. Interestingly, $M$. africanum Lineages 5 and L6 are only limited to West Africa while recent epidemiological work suggests that $M$. tuberculosis Lineage 7 is restricted to Ethiopia [6]. From an evolutionary point of view, it is interesting to note that five out of these seven lineages have been identified in Ethiopia (L1-L4, L7) suggesting a long history of TB in the country [6-8].

Both pulmonary tuberculosis (PTB) and extrapulmonary tuberculosis (EPTB) are significant public health problems in Ethiopia [9]. EPTB is clinically diagnosed or bacteriologically confirmed in organs other than the lungs. Tuberculous lymphadenitis (TBLN) is the most commonly occurring form of EPTB. It manifests as a slowly progressive, painless swelling of lymph nodes, and is caused by strains that are also isolated from cases of PTB including those that are drug resistant [10].

In Ethiopia, comparisons of drug resistance have not been well investigated across the main lineages. The present study was therefore conducted to explore and compare drug resistance patterns among the most prevalent $M$. tuberculosis lineages in Ethiopia including the newly identified L7, using isolates from both PTB and TBLN cases. We analyzed a previously published collection of M. tuberculosis strains from Ethiopia [11] to get insight into drug resistance and MDR-TB rates among the selected isolates.

\section{Methods}

\section{Strain selection}

One hundred and sixty-one $M$. tuberculosis strains used for this study were selected from four lineages from 950 archived isolates that were collected in a cross sectional study during 2006-2010 conducted to identify the disease agents causing PTB and TBLN in Ethiopia [11].
The representation of geographical origins and genotypes with regards to the original study [11] are shown in Additional file 1: Tables S1, Additional file 2: Table S2 and Additional file 3: Table S3. All 950 isolates had previously been characterized as $M$. tuberculosis [11] by RD (Region of Difference) typing and Spoligotyping $[12,13]$ and a subset of these strains had been defined [11] by MIRU-VNTR typing [14].

\section{Drug susceptibility testing}

To explore drug susceptibility among $M$. tuberculosis isolates, the Line-probe assay Genotype MTBDRplus (Hain Lifescience, Germany) was performed according to protocol by manufacturer [15]. This assay is designed to detect strains of the M. tuberculosis complex from clinical specimens and culture, and whether they are resistant to RMP and/or INH.

\section{Data collection}

The data collected by Firdessa et al. 2013 [11] was the baseline data for this work and formulated in Microsoft Access. Data was analyzed using STATA version 11 (Stata Statistical Software: College Station, TX: StataCorp LP).

\section{Results}

\section{Strain selection}

Drug susceptibility testing was undertaken on 161 strains selected from 950 previously characterized and archived M. tuberculosis strains originally isolated from patients in 9 hospitals/health centers in a comprehensive Ethiopian cross sectional study [11]. At large, these 161 M. tuberculosis strains, isolated from 54 TBLN and 107 PTB patients, represented the most common spoligotype(s) from each of four different lineages found in that study and they are listed in the Additional file 1: Table S1. In particular, 41 isolates of SIT25 (L3) and 88 isolates from SIT149 (L4) - the two most frequent spoligotypes in that study - were randomly selected among these two types from the archived strain pool. The percentage of sampled isolates for this study among the total archive from the previously reported survey [11] was $7 / 11$ (63\%) for L1, 41/252 (16\%) for L3, 88/671 (13\%) for L4, and 25/36 (69\%) for L7.

\section{Line probe assay}

All 161 selected isolates were heat-inactivated and tested by GenoType MTBDRplus (v1.0) (Hain Lifescience $\mathrm{GmbH}$, Germany) for Rifampicin (RMP) and Isoniazid (INH) resistance. This line probe assay identified 14 (8.7\%) isolates as resistant to at least one of these two first line anti-tuberculosis drugs. The total number of isolates resistant to INH was 12/161 (7.5\%). The proportion of RMP resistant strains was 4.3\% (7/161) while MDR was detected in $3.1 \%(5 / 161)$ of cases (Table 1$)$. 
Table 1 Drug resistance of Mycobacterium tuberculosis isolates stratified by lineage, Ethiopia ${ }^{a}$

\begin{tabular}{llllll}
\hline Lineage & Number of isolates & Susceptible (\%) & Resistant only to RMP (\%) & Resistant only to INH (\%) & $\begin{array}{l}\text { Resistant to both RMP } \\
\text { and INH (MDR) (\%) }\end{array}$ \\
\hline L1 & 7 & $7(100)$ & 0 & 0 & 0 \\
L3 & 41 & $39(95.2)$ & $1(2.4)$ & $1(2.4)$ & 0 \\
L4 & 88 & $76(86.4)$ & $1(1.1)$ & $6(6.8)$ & $5(5.7)$ \\
L7 & 25 & $25(100)$ & 0 & 0 & 0 \\
All & 161 & $147(91.4)$ & $2(1.2)$ & $7(4.3)$ & $5(3.1)$ \\
\hline
\end{tabular}

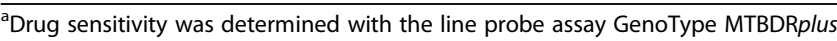

When stratified by disease type, mono-resistance was observed in $3.7 \%(4 / 107)$ of PTB and $7.4 \%(4 / 54)$ of TBLN cases. MDR was detected in 3/107 (2.8\%) PTB and 2/54 (3.7\%) TBLN isolates. When drug resistance was compared among lineages, a higher proportion was observed among L4 strains where 5/88 (5.7\%) were MDR and 7/88 (8.0\%) mono-resistant compared to L3 where there was no MDR strain and only 2/41 (4.9\%) were mono-resistant (Table 1). The number of isolates representing L1 and L7 was low; nevertheless, none of the seven L1 strains and the 25 strains of L7 was resistant to any of the drugs tested.

\section{Drug resistance among SIT149 genotypes}

Most (12/14) of the drug resistant M. tuberculosis isolates identified by the line probe assay in this study belonged to the subset of L4 strains with spoligotype SIT149. These 12 strains come from a subset of 88 SIT149 strains that had previously been characterized by MIRU-VNTR as well [11] leading to further stratification into 24 different genotypes named SIT149:A-Y as shown in Additional file 1: Table S1. Superimposing the genotyping data and the drug resistance results of these 88 isolates showed that the 12 drug resistant strains were clustered to only six genotypes; five strains belonged to SIT149:A, three were of SIT149:L, while the remaining four strains were of genotypes SIT149:B, C, I, and U. In fact, all five strains genotyped as SIT149:A were drug resistant according to the line probe assay (Additional file 1: Table S1), and four of these five strains were actually resistant to both RMP and INH with identical mutation profiles (Table 2); RMP resistance confirmed by mutation "MUT3" (rpoB codon S531 L) and INH resistance by mutation "MUT1" (C15T upstream katG). In comparison, the three drug resistant isolates of genotype SIT149:L (which only diverges from SIT149:A in the number of repeats in MIRU-VNTR locus 2401) showed different mutation profiles (Table 2).

\section{Geographical distribution of SIT149 genotypes}

To get a picture of the geographical distribution of the identified SIT149 genotypes, we mapped all genotypes with two or more M. tuberculosis isolates (Additional file 1:
Table S1; Fig. 1). The most prevalent genotype SIT149:L, which included 31 of 88 strains of spoligotype SIT149, was isolated from patients in most study sites while the other genotypes of SIT149 showed different degrees of geographical clustering. Interestingly, genotype SIT149:A that was shown in this study to have a high rate of drug resistance was only found in Woldiya, Gondar, and Fiche in the Central and Northern Ethiopian highlands. All other identified genotypes were isolated from Central or Southern Ethiopia.

\section{Discussion}

Ethiopia has a long history of human TB [6] and is still hugely affected by the disease and is listed among the TB high-burden countries in the world, with an estimated number of 172,000 new cases (an incidence rate of 164/100,000) in 2017 [1]. Since the introduction of anti-TB drugs in the 1940s, and especially since the 1990s, drug-resistant TB has become a significant threat in the overall fight against TB. For 2017 WHO reported an MDR-TB incidence rate among new TB cases of 3.7\% worldwide and $2.7 \%$ for Ethiopia, i.e. 5500 new cases per year [1].

In the present study, we investigated about $17 \%$ of 950 strains that were collected in one of the largest molecular epidemiology studies of TB in Ethiopia [11]. $M$. tuberculosis isolates of all four lineages that had been identified were selected with particular focus on the two most prevalent spoligotypes, SIT149 (L4) and SIT25 (L3), as well as strains of the recently identified L7. Here we report an overall drug-resistance rate of $8.7 \%$ and an MDR-TB rate of 3.1\% among these strains. A limitation of the study is that the initial population from which the isolates were collected, although extensive and covering several regions of the country, was not randomly selected to be representative of the national population and not all the strains identified were tested for drug resistance. Nevertheless, these figures fall within the range of WHO estimates for Ethiopia [1].

There was a marked difference in rates between lineages. Strains of L1 and L7 were all susceptible to RMP and INH but the number of isolates representing these 
Table 2 Demographic (A), genotyping (B), and drug resistance (C) data of eight Mycobacterium tuberculosis strains of genotypes SIT149:A and SIT149:L

\begin{tabular}{|c|c|c|c|c|c|c|c|c|c|}
\hline \multirow[t]{3}{*}{ A. } & Sample ID & BTBH003 & BTBS101 & BTBS119 & BTBH455 & BTBS785 & BTBS341 & BTBS571 & BTBH946 \\
\hline & Site & Woldiya & Gondar & Fiche & Woldiya & Fiche & Butajira & Woldiya & Butajira \\
\hline & Isolation year & 2006 & 2009 & 2009 & 2007 & 2010 & 2009 & 2010 & 2009 \\
\hline \multirow[t]{25}{*}{ B. } & $G_{\text {Genotype }}^{a}$ & $A$ & $A$ & $A$ & $A$ & A & $L$ & L & L \\
\hline & 580 & 2 & 2 & 2 & 2 & 2 & 2 & 2 & 2 \\
\hline & 2996 & 1 & 1 & 1 & 1 & 1 & 1 & 1 & 1 \\
\hline & 802 & 2 & 2 & 2 & 2 & 2 & 2 & 2 & 2 \\
\hline & 960 & 5 & 5 & 5 & 5 & 5 & 5 & 5 & 5 \\
\hline & 1644 & 1 & 1 & 1 & 1 & 1 & 1 & 1 & 1 \\
\hline & 3192 & 3 & 3 & 3 & 3 & 3 & 3 & 3 & 3 \\
\hline & 424 & 1 & 1 & 1 & 1 & 1 & 1 & 1 & 1 \\
\hline & 577 & 4 & 4 & 4 & 4 & 4 & 4 & 4 & 4 \\
\hline & 2165 & 3 & 3 & 3 & 3 & 3 & 3 & 3 & 3 \\
\hline & 2401 & 5 & 5 & 5 & 5 & 5 & 4 & 4 & 4 \\
\hline & 3690 & 3 & 3 & 3 & 3 & 3 & 3 & 3 & 3 \\
\hline & 4156 & 3 & 3 & 3 & 3 & 3 & 3 & 3 & 3 \\
\hline & $2163 b$ & 1 & 1 & 1 & 1 & 1 & 1 & 1 & 1 \\
\hline & 1955 & 3 & 3 & 3 & 3 & 3 & 3 & 3 & 3 \\
\hline & 4052 & 7 & 7 & 7 & 7 & 7 & 7 & 7 & 7 \\
\hline & 154 & 2 & 2 & 2 & 2 & 2 & 2 & 2 & 2 \\
\hline & 2531 & 5 & 5 & 5 & 5 & 5 & 5 & 5 & 5 \\
\hline & 4348 & 2 & 2 & 2 & 2 & 2 & 2 & 2 & 2 \\
\hline & 2059 & 2 & 2 & 2 & 2 & 2 & 2 & 2 & 2 \\
\hline & 2687 & 1 & 1 & 1 & 1 & 1 & 1 & 1 & 1 \\
\hline & 3007 & 3 & 3 & 3 & 3 & 3 & 3 & 3 & 3 \\
\hline & 2347 & 4 & 4 & 4 & 4 & 4 & 4 & 4 & 4 \\
\hline & 2461 & 2 & 2 & 2 & 2 & 2 & 2 & 2 & 2 \\
\hline & 3171 & 3 & 3 & 3 & 3 & 3 & 3 & 3 & 3 \\
\hline \multirow[t]{16}{*}{$C}$. & $\mathrm{RMP}^{\mathrm{b}}$ & $\mathrm{R}$ & $\mathrm{R}$ & $S$ & $\mathrm{R}$ & $\mathrm{R}$ & $S$ & $\mathrm{R}$ & S \\
\hline & $\mathrm{INH}^{\mathrm{b}}$ & $\mathrm{R}$ & $\mathrm{R}$ & $\mathrm{R}$ & $\mathrm{R}$ & R & $\mathrm{R}$ & $R$ & R \\
\hline & rpoB WT1 & + & + & + & + & + & + & + & + \\
\hline & rpoB WT2 & + & + & + & + & + & + & + & + \\
\hline & rpoB WT3 & + & + & + & + & + & + & + & + \\
\hline & rpoB WT4 & + & + & + & + & + & + & + & + \\
\hline & rpoB WT5 & + & + & + & + & + & + & + & + \\
\hline & rpoB WT6 & + & + & + & + & + & + & + & + \\
\hline & rpoB WT7 & + & + & + & + & + & + & $+^{c}$ & + \\
\hline & rpoB WT8 & - & - & + & - & - & + & $+^{c}$ & + \\
\hline & rpoB Mut1 & - & - & - & - & - & - & - & - \\
\hline & rpoB Mut2A & - & - & - & - & - & - & + & - \\
\hline & rpoB Mut2B & - & - & - & - & - & - & + & - \\
\hline & $\underline{r p o B \text { Mut3 }}$ & + & + & - & + & + & - & + & - \\
\hline & inhA WT1 & + & + & + & + & + & - & $+^{c}$ & - \\
\hline & inhA WT2 & + & + & + & + & + & + & $+^{c}$ & + \\
\hline
\end{tabular}


Table 2 Demographic (A), genotyping (B), and drug resistance (C) data of eight Mycobacterium tuberculosis strains of genotypes SIT149:A and SIT149:L (Continued)

\begin{tabular}{|c|c|c|c|c|c|c|c|c|}
\hline inhA Mut1 & - & - & - & - & - & + & - & + \\
\hline inhA Mut2 & - & - & - & - & - & - & + & - \\
\hline inhA Mut3A & - & - & - & - & - & - & - & - \\
\hline inhA Mut3B & - & - & - & - & - & - & + & - \\
\hline katG WT & - & - & - & - & - & - & - & - \\
\hline katG Mut & + & + & + & + & + & - & - & - \\
\hline katG Mut 2 & - & - & - & - & - & - & - & - \\
\hline
\end{tabular}

Loci with variability between isolates with regards to genotype and drug resistance are underlined and in italic

${ }^{a}$ Genotype refers to Spoligotype SIT149 stratified by number of repeats in respective MIRU-VNTR locus shown here (580; $2996 ; 802 ; 960 ; 1644 ; 3192 ; 424 ; 577$; 2165; 2401; 3690; 4156; 2163b; 1955; 4052; 154; 2531; 4348; 2059; 2687; 3007; 2347; 2461; 3171). A, genotype SIT149:A; L, genotype SIT149:L

${ }^{b} S$ drug susceptible, $R$ drug resistant, + positive hybridization, - negative hybridisation

'Positive hybridisation of wild type probe despite hybridisation to a corresponding mutation; indication of dual infection or cross-contamination of sample

lineages was relatively low, suggesting that representative results may not have been gained at the population level although a relatively higher proportion of samples was tested from the archived isolates for these lineages in this study. The overall prevalence of these lineages is low in the country [11]. Lineage 2, reported to have a tendency to higher frequency of drug resistance [16] is in particular rarely isolated in Ethiopia [7, 8, 11]. Modern and ancient TB lineages have been reported to differ in virulence and their tendency to acquire drug resistance [17]. It can

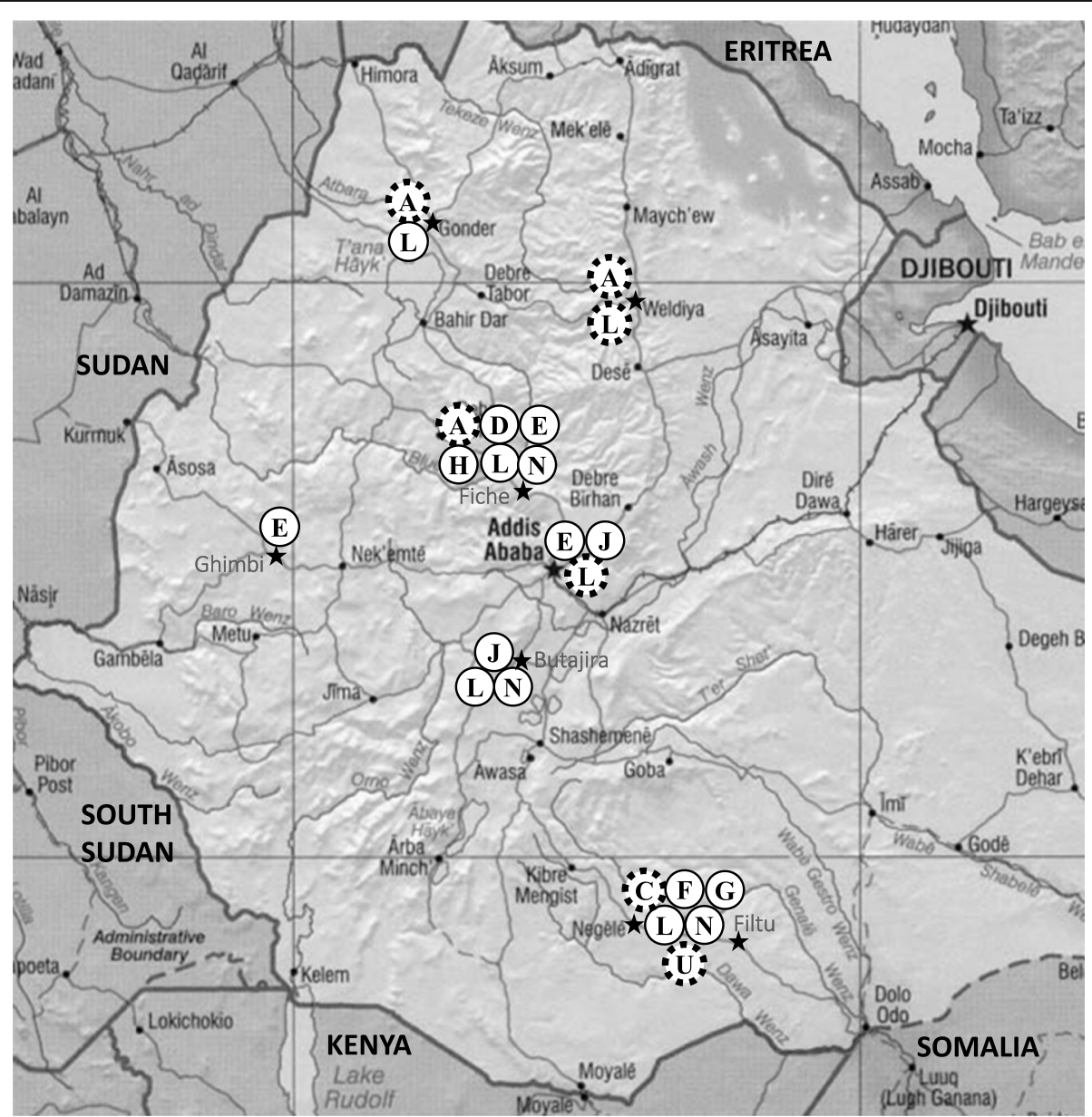

Fig. 1 Geographical distribution of selected genotypes of Mycobacterium tuberculosis shared spoligotype SIT149 in Ethiopia as identified by Firdessa et al. [11]. Study site is marked with a black star. Capitalized letters symbolize genotype of SIT149. Only genotypes with two or more isolates are shown (Additional file 1: Table S1). Dotted line symbolizes genotype with at least one drug resistant isolate identified in that study site 
therefore not be excluded that drug resistance is less frequent among L1 (ancient lineage) and L7, a pre-modern lineage that is rarely found outside Ethiopia [18].

The difference between SIT149 and SIT25 was also noticeable with a higher rate of resistance in SIT149. MDR-TB isolates were only found among genotypes of SIT149 in this study. SIT 149 (also referred to as T3-ETH, ETH-3 [18-20] or more recently as L4.2.ETH1 [6]) is a predominant spoligotype cluster in Ethiopia, more often associated with drug resistance than other spoligotypes in the country [21] and indeed elsewhere, as among Ethiopian immigrants in Israel [22]. Similarly, Agonafir et al. (2010) [23] found SIT149 as the most prevalent and most clustered spoligotype among 46 MDR-TB cases in Addis Ababa, followed by SIT21 and SIT25 (both considered as sub-lineages of L3). However, these observations do not necessarily indicate that these spoligotypes are more prone to be drug-resistant but could rather be a consequence of high prevalence in the population. In fact, a recent review by Panwalkar et al. (2017) [24] summarized many studies in attempts to find correlation between genotypes and TB drug-resistance, but they concluded that it is still uncertain whether there is such an association. On the other hand, it is frequently observed that certain clones emerge from different genotype families and spread rapidly in different populations. The Beijing B0/W148 cluster is a well-known example from Lineage 2 [25]. The Ural family of Lineage 4, with which SIT 149 clusters, although previously considered less transmissible, less virulent and not linked to MDR, has recently been reported to have given rise to MDR strains in Eastern Europe and Russia [26].

In an earlier study, we performed MIRU-VNTR on 88 strains of SIT149 [11] and here we have overlapped that information with the results gained from the line probe assay of the same strains. The most common genotype SIT149:L was identified with $~ 10 \%$ drug resistance (3 out of 31 isolates) of which two isolates were mono-resistant to INH and the third resistant to both INH and RMP (Table 2). In contrast, all five strains of genotype SIT149:A were drug-resistant and four of them (80\%) had an identical resistance profile for INH and RMP in the line probe assay. The latter profile was different to the one found for the MDR-TB strain of SIT149:L. Although the number of isolates of this genotype is fairly low, the observation is important in that it could indicate that a potential MDR-TB clone of genotype SIT149:A is circulating in the Ethiopian highlands. Investigation of currently circulating MDR-TB strains in Ethiopia could explore if this clone is spreading.

\section{Conclusion}

This study examined the rate of RMP and INH resistance among a set of $189 \mathrm{M}$. tuberculosis strains, representing the most common lineages and sub-lineages identified in Ethiopia. The overall drug resistance rate in the series of isolates tested was $8.7 \%$ while the rate of MDR was $3.1 \%$. Although the study design does not allow for generalization since sampling was not based on randomized selection to achieve national representation, and drug resistance was determined with LPA rather than with the gold standard proportional method, the findings do provide insight into existing drug resistance trends in the country. Moreover, we have identified a sub-lineage of $M$. tuberculosis L4 that showed a high rate of resistance to both RMP and INH. These drug resistant strains may belong to a clone of $M$. tuberculosis that is circulating in the highlands of Ethiopia.

\section{Additional files}

Additional file 1: Table S1. Summary of strain characteristics for 161 Mycobacterium tuberculosis isolates included in this study. (XLSX $28 \mathrm{~kb}$ )

Additional file 2: Table S2. Delineation of isolates used in this study. (XLSX $12 \mathrm{~kb}$ )

Additional file 3: Table S3. Strain selection in relation to previous survey. (DOCX $13 \mathrm{~kb}$ )

\section{Abbreviations \\ INH: Isoniazid; MDR: Multi-drug resistant; MIRU-VNTR: Mycobacterial Interspersed Repetitive Unit - Variable Number Tandem Repeat; PTB: Pulmonary TB; RMP: Rifampicin; SIT: Shared international type; TB: Tuberculosis; TBLN: TB lymphadenitis in the cervical lymph node}

\section{Acknowledgements}

Our appreciation goes to staff at the Armauer Hansen Research Institute (AHRI) who provided considerable support.

\section{Funding}

This study was funded by the Wellcome Trust (grant 075833/A/04/Z), under the Animal Health in the Developing World initiative, by AHRI from its Norad and Sida core grant and by Addis Ababa University. AA receives support from NIH Fogarty International Center (award number D43TW009127). The funding bodies had no role in the study design and collection, analysis, and interpretation of data and in writing the manuscript.

Availability of data and materials

All pertinent data are included in the manuscript and its supplementary files.

\section{Authors' contributions}

Experimental design: ShB, YD, StB, AA. Experimental performance (lab work): ShB, YD, EH. Analyzing the results: ShB, YD, StB, TH, SA. Writing the manuscript: StB, ShB, AA, YD, LY, DB, AM, SA, KD. Approved manuscript for submission: ShB, YD, StB, AA, EH, LY, TH, KD, AM, SA, DB. All authors have read and agreed for publication of the manuscript.

\section{Ethics approval and consent to participate}

Ethical approvals for this study were obtained from AHRI/ALERT Ethical Review Committee and the Ethics Committee of the Department of Microbial, Cellular and Molecular Biology, Faculty of Natural Sciences, Addis Ababa University. Written informed consent had been obtained from each participant in the original study from which the archived isolates were retrieved. Strict confidentiality of all isolates was maintained and no personal identifiers were used.

Consent for publication Not applicable. 


\section{Competing interests}

The authors declare that they have no competing interests.

\section{Publisher's Note}

Springer Nature remains neutral with regard to jurisdictional claims in published maps and institutional affiliations.

\section{Author details}

'Armauer Hansen Research Institute, Jima Road, Addis Ababa, Ethiopia. 2Department of Microbial, Cellular and Molecular Biology, College of Natural Sciences, Addis Ababa University, Addis Ababa, Ethiopia. ${ }^{3}$ Animal and Plant Health Agency, New Haw, Surrey, UK. ${ }^{4}$ Present address: J. Craig Venter Institute, Rockville, MD, USA.

\section{Received: 6 September 2018 Accepted: 25 November 2018}

\section{Published online: 04 December 2018}

\section{References}

1. Global tuberculosis report 2018 [www.who.int/tb/data]. Accessed 30 Nov 2018.

2. Borrell S, Gagneux S. Infectiousness, reproductive fitness and evolution of drug-resistant Mycobacterium tuberculosis. Int J Tuberc Lung Dis. 2009;13(12):1456-66

3. Chung-Delgado K, Guillen-Bravo S, Revilla-Montag A, Bernabe-Ortiz A Mortality among MDR-TB cases: comparison with drug-susceptible tuberculosis and associated factors. PLoS One. 2015:10(3):e0119332.

4. Ling DI, Zwerling AA, Pai M. Rapid diagnosis of drug-resistant TB using line probe assays: from evidence to policy. Expert review of respiratory medicine. 2008;2(5):583-8.

5. van Klingeren B, Dessens-Kroon M, van der Laan T, Kremer K, van Soolingen D. Drug susceptibility testing of Mycobacterium tuberculosis complex by use of a high-throughput, reproducible, absolute concentration method. J Clin Microbiol. 2007:45(8):2662-8.

6. Comas I, Hailu E, Kiros T, Bekele S, Mekonnen W, Gumi B, Tschopp R, Ameni $\mathrm{G}$, Hewinson RG, Robertson BD, et al. Population genomics of Mycobacterium tuberculosis in Ethiopia contradicts the virgin soil hypothesis for human tuberculosis in sub-Saharan Africa. Curr Biol. 2015:25(24):3260-6.

7. Gumi B, Schelling E, Berg S, Firdessa R, Erenso G, Mekonnen W, Hailu E, Melese E, Hussein J, Aseffa A, et al. Zoonotic transmission of tuberculosis between pastoralists and their livestock in south-East Ethiopia. EcoHealth. 2012:9(2):139-49.

8. Ali S, Beckert P, Haileamlak A, Wieser A, Pritsch M, Heinrich N, Loscher T, Hoelscher M, Niemann S, Rachow A. Drug resistance and population structure of $M$. tuberculosis isolates from prisons and communities in Ethiopia. BMC Infect Dis. 2016;16(1):687.

9. Biadglegne F, Tesfaye W, Anagaw B, Tessema B, Debebe T, Anagaw B, Mulu A, Sack U, Rodloff AC. Tuberculosis lymphadenitis in Ethiopia. Jpn J Infect Dis. 2013:66(4):263-8.

10. Fontanilla J-M, Barnes A, Von Reyn CF. Current diagnosis and management of peripheral tuberculous lymphadenitis. Clin Infect Dis. 2011;53(6):555-62

11. Firdessa R, Berg S, Hailu E, Schelling E, Gumi B, Erenso G, Gadisa E, Kiros T, Habtamu M, Hussein J, et al. Mycobacterial lineages causing pulmonary and extrapulmonary tuberculosis, Ethiopia. Emerg Infect Dis. 2013:19(3):460-3.

12. Brosch R, Gordon SV, Marmiesse M, Brodin P, Buchrieser C, Eiglmeier K, Garnier T, Gutierrez C, Hewinson G, Kremer K, et al. A new evolutionary scenario for the Mycobacterium tuberculosis complex. Proc Natl Acad Sci U S A. 2002:99(6):3684-9.

13. van Embden JD, van Gorkom T, Kremer K, Jansen R, van Der Zeijst BA, Schouls LM. Genetic variation and evolutionary origin of the direct repeat locus of Mycobacterium tuberculosis complex bacteria. J Bacteriol. 2000;182(9):2393-401.

14. Allix-Beguec C, Fauville-Dufaux M, Supply P. Three-year population-based evaluation of standardized mycobacterial interspersed repetitive-unitvariable-number tandem-repeat typing of Mycobacterium tuberculosis. J Clin Microbiol. 2008;46(4):1398-406.

15. Hillemann D, Weizenegger M, Kubica T, Richter E, Niemann S. Use of the genotype MTBDR assay for rapid detection of rifampin and isoniazid resistance in Mycobacterium tuberculosis complex isolates. J Clin Microbiol. 2005:43(8):3699-703.
16. Sarkar R, Lenders L, Wilkinson KA, Wilkinson RJ, Nicol MP. Modern lineages of Mycobacterium tuberculosis exhibit lineage-specific patterns of growth and cytokine induction in human monocyte-derived macrophages. PLoS One. 2012;7(8):e43170.

17. Gagneux S. Host-pathogen coevolution in human tuberculosis. Philos Trans R Soc Lond Ser B Biol Sci. 2012;367(1590):850-9.

18. Yimer SA, Namouchi A, Zegeye ED, Holm-Hansen C, Norheim G, Abebe M, Aseffa A, Tonjum T. Deciphering the recent phylogenetic expansion of the originally deeply rooted Mycobacterium tuberculosis lineage 7. BMC Evol Biol. 2016;16(1):146.

19. Belay M, Ameni G, Bjune G, Couvin D. Strain diversity of Mycobacterium tuberculosis isolates from pulmonary tuberculosis patients in Afar pastoral region of Ethiopia. Biomed Res Int. 2014;2014:238532.

20. Brudey K, Driscoll JR, Rigouts L, Prodinger WM, Gori A, Al-Hajoj SA, Allix C, Aristimuno L, Arora J, Baumanis V, et al. Mycobacterium tuberculosis complex genetic diversity: mining the fourth international spoligotyping database (SpolDB4) for classification, population genetics and epidemiology. BMC Microbiol. 2006;6:23.

21. Yimer SA, Norheim G, Namouchi A, Zegeye ED, Kinander W, Tonjum T, Bekele S, Mannsaker T, Bjune G, Aseffa A, et al. Mycobacterium tuberculosis lineage 7 strains are associated with prolonged patient delay in seeking treatment for pulmonary tuberculosis in Amhara region, Ethiopia. J Clin Microbiol. 2015;53(4):1301-9.

22. Freidlin PJ, Nissan I, Luria A, Goldblatt D, Schaffer L, Kaidar-Shwartz H, Chemtob D, Dveyrin Z, Head SR, Rorman E. Structure and variation of CRISPR and CRISPR-flanking regions in deleted-direct repeat region Mycobacterium tuberculosis complex strains. BMC Genomics. 2017;18(1):168.

23. Agonafir M, Lemma E, Wolde-Meskel D, Goshu S, Santhanam A, Girmachew F, Demissie D, Getahun M, Gebeyehu M, van Soolingen D. Phenotypic and genotypic analysis of multidrug-resistant tuberculosis in Ethiopia. Int J Tuberc Lung Dis. 2010;14(10):1259-65.

24. Panwalkar N, Chauhan DS, Desikan P. Spoligotype defined lineages of Mycobacterium tuberculosis and drug resistance: merely a casual correlation? Indian J Med Microbiol. 2017;35(1):27-32.

25. Mokrousov I. Insights into the origin, emergence, and current spread of a successful Russian clone of Mycobacterium tuberculosis. Clin Microbiol Rev. 2013;26(2):342-60.

26. Mokrousov I. Emerging clones of Mycobacterium tuberculosis in Russia and former Soviet Union countries: Beijing genotype and beyond. Int J Mycobacteriol. 2016;5(Suppl 1):S69-s70.

\section{Ready to submit your research? Choose BMC and benefit from:}

- fast, convenient online submission

- thorough peer review by experienced researchers in your field

- rapid publication on acceptance

- support for research data, including large and complex data types

- gold Open Access which fosters wider collaboration and increased citations

- maximum visibility for your research: over $100 \mathrm{M}$ website views per year

At $\mathrm{BMC}$, research is always in progress.

Learn more biomedcentral.com/submission 